\title{
Crystal structure of signal regulatory protein gamma (SIRP $\gamma$ ) in complex with an antibody Fab fragment
}

Joanne E Nettleship ${ }^{1,2+}$, Jingshan Ren ${ }^{1 \dagger}$, David J Scott ${ }^{3,4}$, Nahid Rahman ${ }^{1,2}$, Deborah Hatherley ${ }^{5}$, Yuguang Zhao ${ }^{1}$, David I Stuart ${ }^{1,6}$, A Neil Barclay ${ }^{5}$ and Raymond J Owens ${ }^{1,2^{*}}$

\begin{abstract}
Background: Signal Regulatory Protein $\gamma(\mathrm{SIRP} \gamma)$ is a member of a closely related family of three cell surface receptors implicated in modulating immune/inflammatory responses. SIRPY is expressed on T lymphocytes where it appears to be involved in the integrin-independent adhesion of lymphocytes to antigen-presenting cells. Here we describe the first full length structure of the extracellular region of human SIRPY.

Results: We obtained crystals of SIRPy by making a complex of the protein with the Fab fragment of the anti-SIRP antibody, OX117, which also binds to SIRPa and SIRP 3 . We show that the epitope for FabOX117 is formed at the interface of the first and second domains of SIRPY and comprises residues which are conserved between all three SIRPs. The FabOX 117 binding site is distinct from the region in domain 1 which interacts with CD47, the physiological ligand for both SIRPY and SIRPa but not SIRP $\beta$. Comparison of the three domain structures of SIRPY and SIRPa showed that these receptors can adopt different overall conformations due to the flexibility of the linker between the first two domains. SIRPY in complex with FabOX117 forms a dimer in the crystal. Binding to the Fab fixes the position of domain 1 relative to domains 2/3 exposing a surface which favours formation of a homotypic dimer. However, the interaction appears to be relatively weak since only monomers of SIRPY were observed in sedimentation velocity analytical ultracentrifugation of the protein alone. Studies of complex formation by equilibrium ultracentrifugation showed that only a 1:1 complex of SIRPY: FabOX117 was formed with a dissociation constant in the low micromolar range $\left(K_{d}=1.2+/-0.3 \mu \mathrm{M}\right)$.
\end{abstract}

Conclusion: The three-domain extracellular regions of SIRPs are structurally conserved but show conformational flexibility in the disposition of the amino terminal ligand-binding lg domain relative to the two membrane proximal Ig domains. Binding of a cross-reactive anti-SIRP Fab fragment to SIRPY stabilises a conformation that favours SIRP dimer formation in the crystal structure, though this interaction does not appear sufficiently stable to be observed in solution.

Keywords: Antigen-binding complex, Signal regulatory protein, Receptor structure

\footnotetext{
*Correspondence: ray@strubi.ox.ac.uk

${ }^{\dagger}$ Equal contributors

'Division of Structural Biology, Henry Wellcome Building for Genomic

Medicine, University of Oxford, Roosevelt Drive, Oxford OX3 7BN, UK

${ }^{2} \mathrm{OPPF}-U K$, The Research Complex at Harwell, Rutherford Appleton

Laboratory, Harwell Oxford, Oxfordshire OX11 OFA, UK

Full list of author information is available at the end of the article
}

\section{Biomed Central}

(c) 2013 Nettleship et al.; licensee BioMed Central Ltd. This is an Open Access article distributed under the terms of the Creative Commons Attribution License (http://creativecommons.org/licenses/by/2.0), which permits unrestricted use distribution, and reproduction in any medium, provided the original work is properly cited. 


\section{Background}

Members of the signal regulatory protein family (SIRP) play important roles in the regulation of the immune response in man [1]. The family comprises three type I transmembrane glycoproteins $(\alpha, \beta, \gamma)$ each with an extracellular region consisting of three Ig-like domains followed by a single transmembrane sequence and a cytoplasmic domain which varies in length between the three SIRPs. SIRPs are classified as "paired receptors" since they show the following characteristics: (1) they are encoded by different genes arranged in a gene cluster, (2) they share significant sequence homology in their extracellular domains and (3) they comprise both activating and inhibitory members. Thus SIRPa delivers an inhibitory signal via immunoreceptor tyrosine-based inhibition motifs (ITIMs) located in the cytoplasmic domain of the protein as well as interacting with the ligand CD47 [2]. SIRP $\beta$ delivers an activating signal through association with DAP12, a transmembrane adaptor protein with an immunoreceptor tyrosinebased activation motif (ITAM), but does not bind to CD47 [1]. By contrast, SIRPY appears to have no signaling function but does bind to CD47, though this interaction is ten times weaker than that of SIRP $\alpha$ [3]. Unlike the other SIRP proteins, SIRPy is expressed by T cells where it interacts with CD47 on the surface of the cell, resulting in increased cell-cell adhesion in an integrin-independent manner [4]. Therefore, it is thought that SIRPY may be involved in $\mathrm{T}$ cell responses as an accessory protein [4]. Note, SIRP $\gamma$ was originally called SIRP $\beta 2$ but this term is no longer used [5].

There has been considerable interest in the structure of the SIRP family with regard to the subtle differences in ligand binding specificity $[6,7]$ and a putative relationship to primitive antigen receptors [8]. Crystal structures have been determined for the $\mathrm{N}$ terminal domains of SIRP $\alpha$ [9,10], SIRP $\beta$ and SIRP $\gamma$ [11], a full extracellular region of SIRP $\alpha$ [12] and a complex of the N-terminal domain of SIRP $\alpha$ and CD47 [11].

In this paper we present the X-ray crystal structure of the complete extracellular portion of SIRP $\gamma$ co-crystallized with the Fab fragment of the OX117 monoclonal antibody, which recognises SIRP $\alpha$, SIRP $\beta$ and SIRP $\gamma$ [3]. The Fab fragments of antibodies have been used extensively as cocrystallization chaperones $[13,14]$ and binding to Fab fragments of OX117 facilitated the crystallization of SIRPY in this study. The eptiope recognised by OX117 involved residues in both domains 1 and 2 ( $\mathrm{d} 1$ and $\mathrm{d} 2$ ) of SIRPY and was distinct from the CD47 binding site on d1. Interestingly, SIRPY formed a dimer in the crystal structure through an interface between $\mathrm{d} 1$ and $\mathrm{d} 2$.

\section{Results and discussion Overall structure}

In contrast to SIRPa [12], attempts to grow diffraction quality crystals of the full three domain extracellular region of SIRPY proved unsuccessful. Therefore an antibody chaperone approach was adopted using the Fab fragment of a SIRP monoclonal antibody (OX117) that had previously been generated $[3,15]$. Both Fab fragments of OX117 and the extracellular region of SIRPY were produced as recombinant proteins in mammalian cells. Diffracting crystals of the FabOX117: SIRPy complex were successfully obtained following de-glycosylation of the SIRPY: FabOX117 protein complex using endoglycosidase F1. The structure of the complex was solved to $2.5 \AA$ resolution by molecular replacement. The crystallographic asymmetric unit contains a SIRP $\gamma$ dimer, with each SIRPY monomer bound to a FabOX117 (Figure 1A). The final refined model has a R-factor of 0.200 (R-free: 0.259 ) with root mean square deviations (rmsds) of $0.007 \AA$ for bond lengths and $1.2^{\circ}$ for bond angles from the ideal values. The C-terminal end of $\mathrm{d} 3$ of SIRPY is flexible, residues 294-295 and 317-332 in one molecule, and residues 232-239, 289-298 and 316-332 in another are not defined in the electron density map and not included in the model. The two SIRPY molecules can be superimposed with a rmsd of $0.61 \AA$ for 294 equivalent $C \alpha$ atoms, while the rmsd is $0.63 \AA$ for 428 equivalent $\mathrm{C} \alpha$ atoms between the two FabOX117 molecules. Of the 4 potential glycosylation sites in each SIRPY molecule, one at residue 240 has sufficient density allowing the N-linked $\mathrm{N}$-acetylglucosamine to be modelled; weak electron density attached to residue 215 suggests the presence of glycosylation. There is no evidence in the electron density map for the modification at Asn281 and Asn289, which contrasts with the observations reported for the structure of SIRP $\alpha$ [12]. The C-terminal $\mathrm{His}_{6}$ tag from one of the Fab heavy chains is ordered, presumably due to crystal contacts. Two His residues, at position 1 and 5, together with a water molecule and His56 from a symmetry related SIRPy bind a zinc ion with the canonical tetrahedral coordination.

\section{The Fab binding site}

From the structure it can be seen that the antibody Fab fragment binds to both $\mathrm{d} 1$ and $\mathrm{d} 2$ of SIRPY (Figure 1A). The light chain variable region $(\mathrm{Vl})$ appears to be inserted between the two domains contacting residues in both $\mathrm{d} 1$ and $\mathrm{d} 2$, whereas the heavy chain variable domain (Vh) only interacts with d1. Comparison of bound and unbound FabOX117 (PDB id 3DIF) shows only small changes in conformation upon binding (Figure 1B). Alignment using PDBeFold [16] of the Fd region (Vh and $\mathrm{CH} 1$ domains) gives a rmsd of $0.6 \AA$ for the $\mathrm{C} \alpha$ backbone for 208 out of 220 residues. Regions of the Fd fragment that were disordered in the unbound Fab structure, namely the Vh loop consisting of residues G133 to S140 and the C-terminal residues 219 to 220 become ordered on binding to the antigen. For the light 


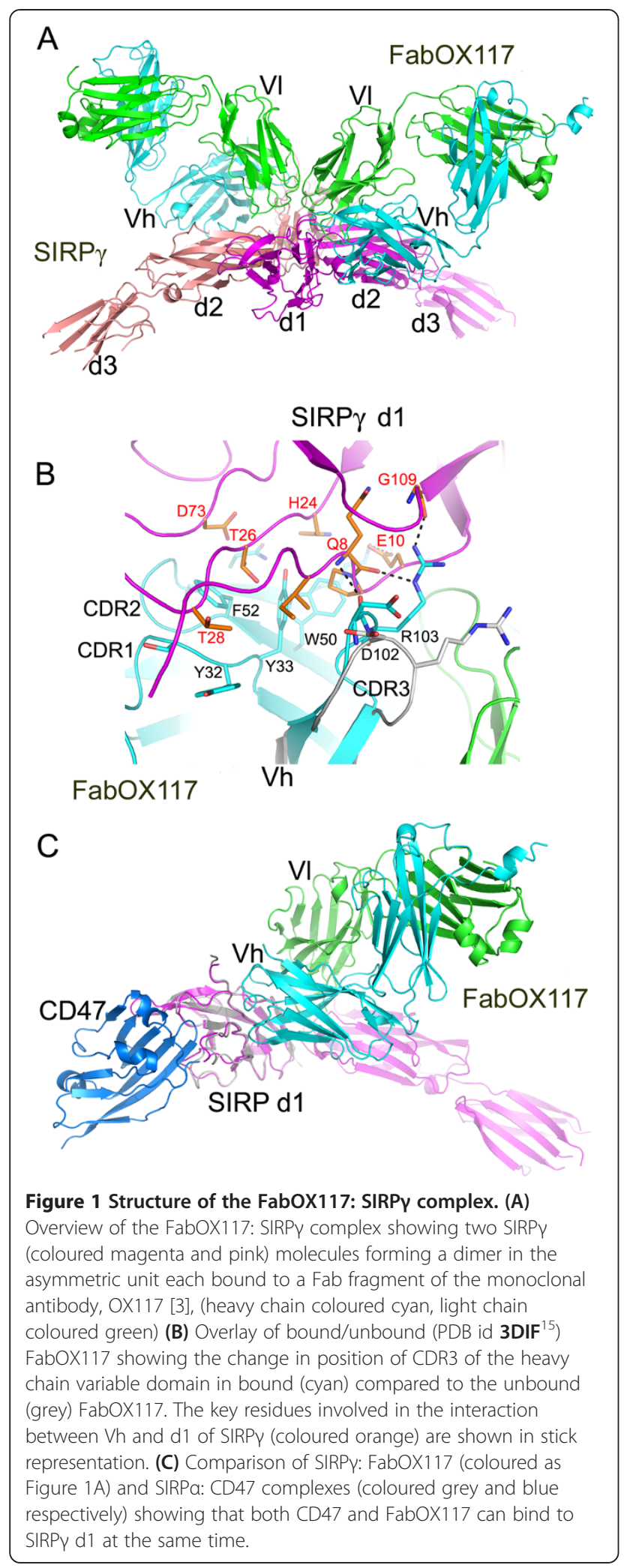

chain the rmsd for the C $\alpha$ backbone is $0.7 \AA$ for 213 out of 214 residues. The most significant conformational change in FabOX117 upon binding to SIRPY is the repositioning of the side-chain of R103 in the third Complementarity Determining Region (CDR3) of Vh (Figure 1B). R103 forms a network of hydrogen bonds with SIRPy d1 involving Q8 and E10 in the loop connecting $\beta$-strands A1 and A2 and G109 in the G1/G2 loop (Figure 1B). Additional hydrogen bond interactions between FabOX117 and SIRPY are observed between Y92 in Vl CDR3 and K11 and L12 in the A1/A2 loop of SIRPY and between S26 of Vl CDR1 and D149 in d2 of SIRPy. All these residues are conserved between SIRP $\alpha, \beta$ and $\gamma$ indicating that OX117 would bind in a similar way to all three SIRPs, consistent with the cross-reactivity data previously published for this antibody [3].

The structure of the isolated d1 domain of SIRPY has been solved previously (PDB id 2JJW [11]). Overlaying this structure onto the full length SIRP $\gamma$, showed that binding of the Fab fragment to d1 did not change the conformation of the domain. Thus alignment of the two d1 structures gave a rmsd for the C $\alpha$ backbone of $0.5 \AA$ for 109/116 amino acids, with the loop comprising G97 to E100 becoming ordered in the complex. Overall, binding of the FabOX117 to SIRPY appears to result in very little conformational change in either the antibody or antigen.

The crystal structure of $\mathrm{d} 1$ of SIRP $\alpha$ in complex with CD47 has been solved by Hatherley et al. [11]. When the $\mathrm{d} 1$ of the FabOX117: SIRPy crystal structure is aligned with the SIRP $\alpha(\mathrm{d} 1)$ : CD47 structure (PDB id 2JJS), the locations where Fab and CD47 bind to SIRPY do not overlap (Figure $1 \mathrm{C}$ ). The main contact residues for CD47 are in three loops connecting strands B2-C, D-E and F-G1. This suggests that SIRPY could bind both FabOX117 and CD47 at the same time.

\section{Comparison of SIRPY with SIRPa}

Comparison of the full length extracellular three domain structure of SIRPy with that of SIRPa (PDB id 2WNG [12]) shows a high level of structural similarity for the individual domains (d1-d3) (Figure 2A). One small difference is the relative length of the C' and D strands in the $\mathrm{d} 3$ domains which are short and long respectively in SIRP $\gamma$ and the opposite in SIRP $\alpha$, though this does not alter the overall topology of the $\mathrm{d} 3$ domains (Figure 2B). However, there is a significant difference in the relative orientation of the three domains between the two SIRP structures (Figure 2A). Thus, in the superimposition of d1 of SIRPY onto the d1 of SIRP (rmsd of $0.60 \AA$ for $105 \mathrm{C} \alpha$ atoms out of 114 residues), the $\mathrm{d} 2-\mathrm{d} 3$ domains of the two proteins are rotated by $66^{\circ}$ with respect to each other (Figure 2A). The difference in the relative arrangement of $\mathrm{d} 1$ and $\mathrm{d} 2-\mathrm{d} 3$ in the two SIRPs would 


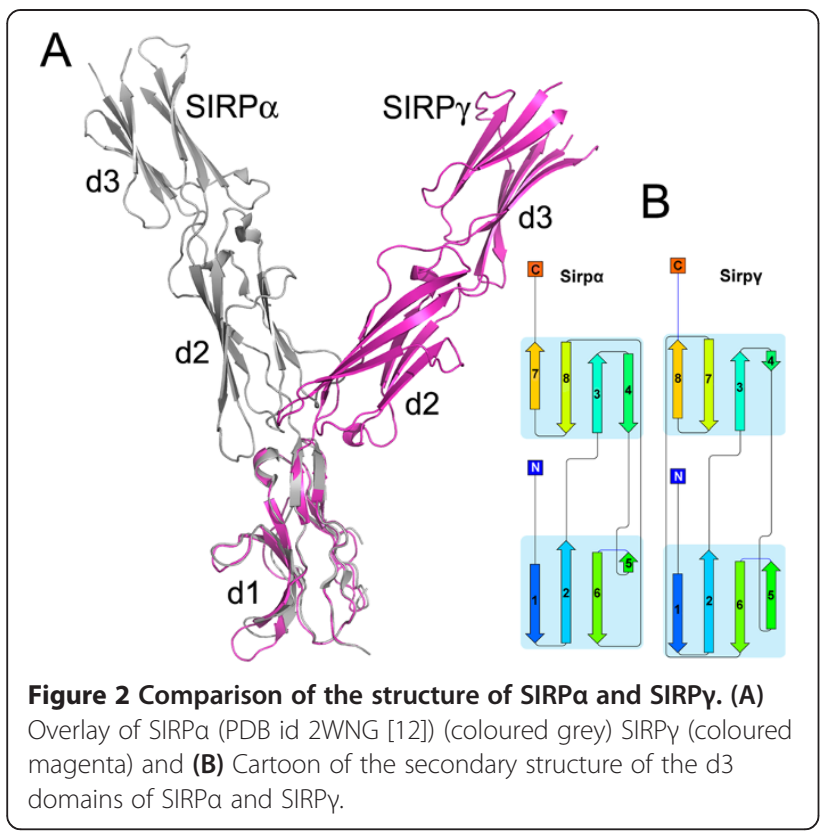

affect the ability of SIRP $\alpha$ to bind to FabOX117 since the interactions between FabOX117 VL and d2 observed in SIRP $\gamma$ would not be possible in the SIRP $\alpha$ structure shown in Figure 2 (PDB id 2WNG). This suggests that there is some flexibility in the d1-d2 inter-domain region as OX117 has previously been shown to bind to all three SIRPs [3].

Interestingly, in contrast to the structure of SIRP $\alpha$ alone [12], the two SIRPy molecules bound to FabOX117 formed a head-to head dimer in the crystal structure (Figure 1A) with a contact area of about $1130 \AA^{2}$ and a Complex Formation Significance Score [17] of 0.78. This is at the lower end of the range considered to be physiological significant $\left(1600+/-400 \AA^{2}\right)$ [18] though it is comparable to the contact surface area $\left(1150 \AA^{2}\right)$ and significance score (0.74) for the SIRPY: Fab interface. It therefore appears to be non-trivial. The two SIRPY molecules form an arch-like structure spanning a distance of approximately $160 \AA$ with the Fab fragments binding to the outer surface of the arch (Figure 3A). Dimer formation appears to be a consequence of the relative orientation of $\mathrm{d} 1$ with respect to $\mathrm{d} 2$ which is imposed by the Fab binding. The interface between the two SIRPY chains comprises a number of hydrogen-bond interactions between residues in $\mathrm{d} 1$ and d2, e.g. T88-D169, L114-S174, E111-G172 and a salt bridge R180-E47 (Figure 3B). Six of these interface residues are conserved between all three SIRP sequences, with the other two showing relatively conservative substitutions (R180H and L114V).

\section{Analysis of the SIRPY: FabOX117 complex by analytical ultracentrifugation}

The observation that SIRPY is dimeric in the crystal, poses the question as to whether this self-association occurs in

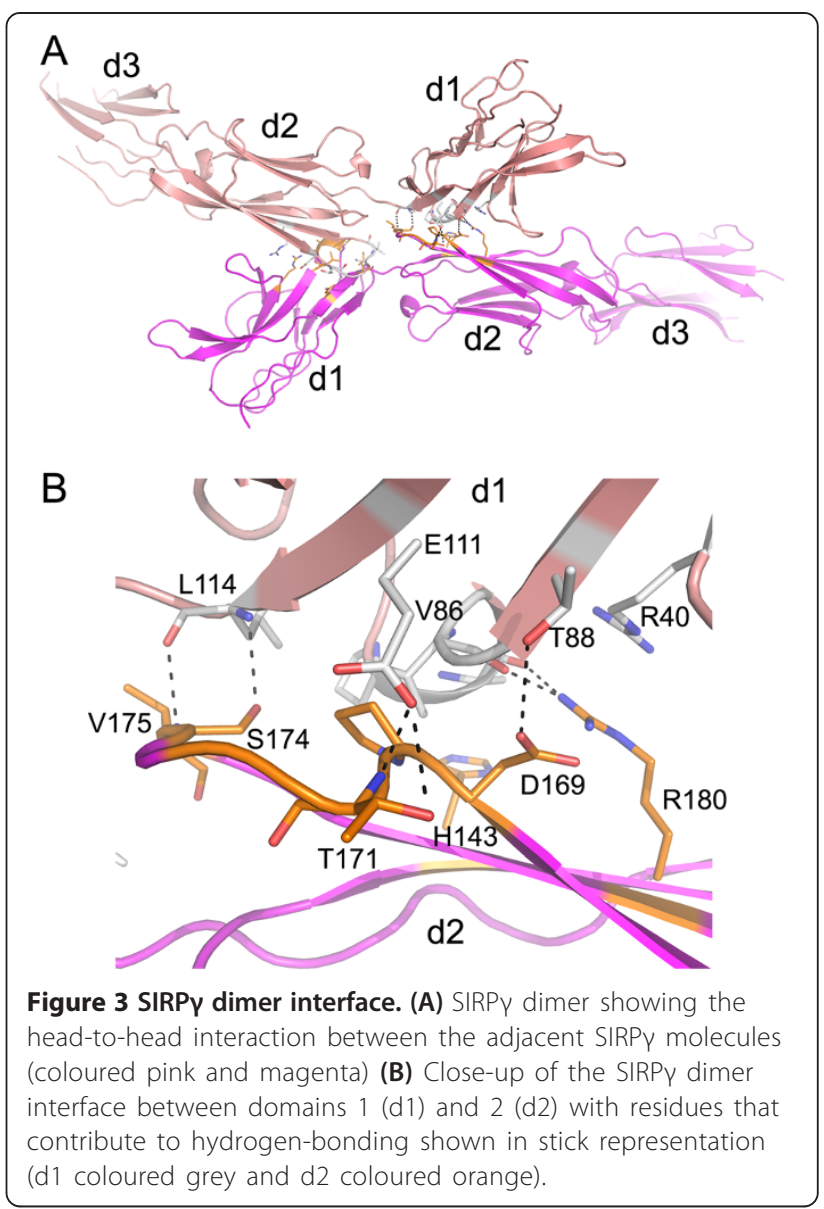

solution. Sedimentation velocity analytical ultracentrifugation experiments were carried out on both SIRPY and FabOX117 proteins individually. Experimentally determined sedimentation coefficients, frictional ratios and molecular weights were compared to values calculated from the coordinates of their crystal structures using USSOMO [19] (Table 1). The results showed that SIRPY was monodisperse and behaved as a monomer. A single species was also observed for FabOX117 which corresponded to the assembled light and heavy chain. Sedimentation velocity experiments of a 2:1 stoichiometric mixture of SIRPY: FabOX117 gave two peaks at $2.70 \mathrm{~S}$ and $4.85 \mathrm{~S}$ (Figure 4A). The first peak was consistent with the calculated and measured value for SIRPY alone, whilst the second peak corresponded to the sedimentation coefficient calculated for a 1:1 complex of SIRP $\gamma$ and FabOX117 (Table 1; marked 'A' in Figure 4A). A 1:2 mixture of SIRPY and FabOX117 gave a single skewed distribution around a sedimentation coefficient of $4.5 \mathrm{~S}$ (Figure 4A), close to that determined for the 1:1 complex. We interpret this result as follows: under the conditions where there is a two-fold molar excess of the FabOX117 over the SIRPY protein, a 1:1 complex with SIRPY is formed, which partially dissociates during sedimentation. Since the free FabOX117 has a 
Table 1 Hydrodynamic parameters

\begin{tabular}{|c|c|c|c|c|c|c|}
\hline Sample & $M_{a p p}^{0}{ }^{a}$ & $S_{20, w}^{0}{ }^{b}$ & $\left(\frac{f}{f_{0}}\right)_{a p p} b$ & $M_{\text {calc }}{ }^{c}$ & $S_{\text {calc }}{ }^{c}$ & $\left(\frac{f}{f_{o}}\right)_{\text {calc }}{ }^{c}$ \\
\hline Fab & 51.2 & 3.7 & 1.4 & 48.4 & 3.81 & 1.22 \\
\hline Sirp & 39.4 & 2.7 & 1.6 & 42.6 & 2.52 & 1.46 \\
\hline 1:1 Complex & $91.0^{d}$ & 4.8 & 1.5 & 91.0 & 4.85 & 1.42 \\
\hline 2:2 Complex & $\mathrm{Nd}$ & $\mathrm{Nd}$ & $\mathrm{Nd}$ & 182.0 & 7.25 & 1.48 \\
\hline
\end{tabular}

${ }^{a}$ calculated using SEDANAL. Units are in kilodaltons.

b determined from sedimentation velocity. Units are in S.

ccalculated from the PDB file using US-SOMO. Units are the same for the observed parameters.

Nd not determined.

similar sedimentation coefficient to the FabOX117: SIRPY complex ( $4.85 \mathrm{~S}$ compared to $4.5 \mathrm{~S}$ ) there is no resolution of the bound and unbound FabOX117 into individual peaks, and a skewed composite peak observed. However the sedimentation coefficient of SIRPY alone is sufficiently different from the complex ( $2.5 \mathrm{~S}$ vs. $4.5 \mathrm{~S})$ that a peak corresponding to the SIRPY alone can be resolved (Figure 4A). The theoretical basis for this behaviour has been described previously by Cann, Winzor and co-workers [20,21]). Nonetheless, if the SIRPY: FabOX117 formed a 2:2 complex, as observed in the crystal structure, then a species sedimenting at $7.25 \mathrm{~S}$ would be expected (see Table 1; marked 'B' in Figure 4A). However no such higher order species were observed in any of the sedimentation velocity experiments.

To assess the strength of the SIRPY: FabOX117 interaction, sedimentation equilibrium centrifugation experiments were carried out on 2:1, 1:1 and 1:2 stoichiometric mixtures of SIRPY: FabOX117. Data were extracted using SEDFIT v14.1 [22] and processed with SEDPHAT [23]. The results did not fit a single species model indicating that multiple forms were present in solution (Figure 4B). However, the data fitted well to an $\mathrm{A}+\mathrm{B} \leftrightarrow \mathrm{AB}$ model. From this model the derived dissociation constant was determined to be $1.2( \pm 0.3) \mu \mathrm{M}$, indicating only a moderately strong antibody: antigen interaction. Such a value would also explain the behaviour of the sedimentation velocity profiles seen in Figure 4A, since at the loading concentration used, there would be a small amount of dissociation into individual components giving rise to skewed peaks in the c(s) plot (Figure 4A).

We conclude that the 2:2 SIRP: FabOX117 complex observed in the crystal structure is not formed in solution under the conditions of the AUC experiments. It is important to note that the highest protein concentration of the SIRPY: FabOX117 mixture used in the AUC experiments was $1 \mathrm{mg} / \mathrm{ml}$ (corresponding to $13.7 \mu \mathrm{M}$ ) compared to $16.8 \mathrm{mg} / \mathrm{ml}(230 \mu \mathrm{M})$ for crystallization. Therefore it is possible that the 2:2 complex predominates at high concentrations of the SIRPY FabOX117 mixture. However we calculate that this would require that the dissociation constant of the $2: 2$ complex is $</=10 \mu \mathrm{M}$. If this was the case

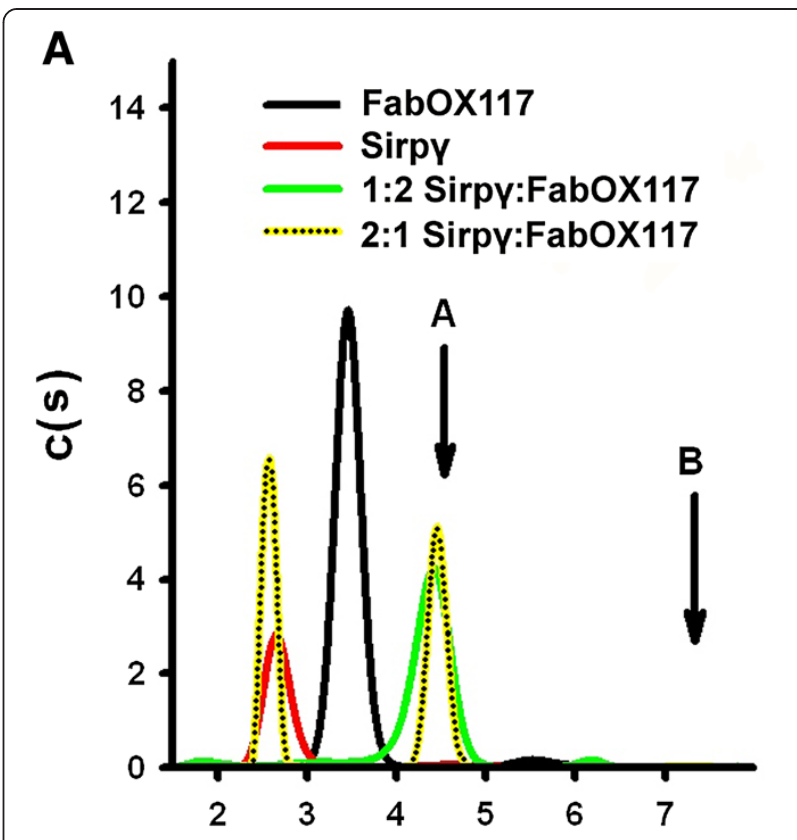

Sedimentation coefficient(S)

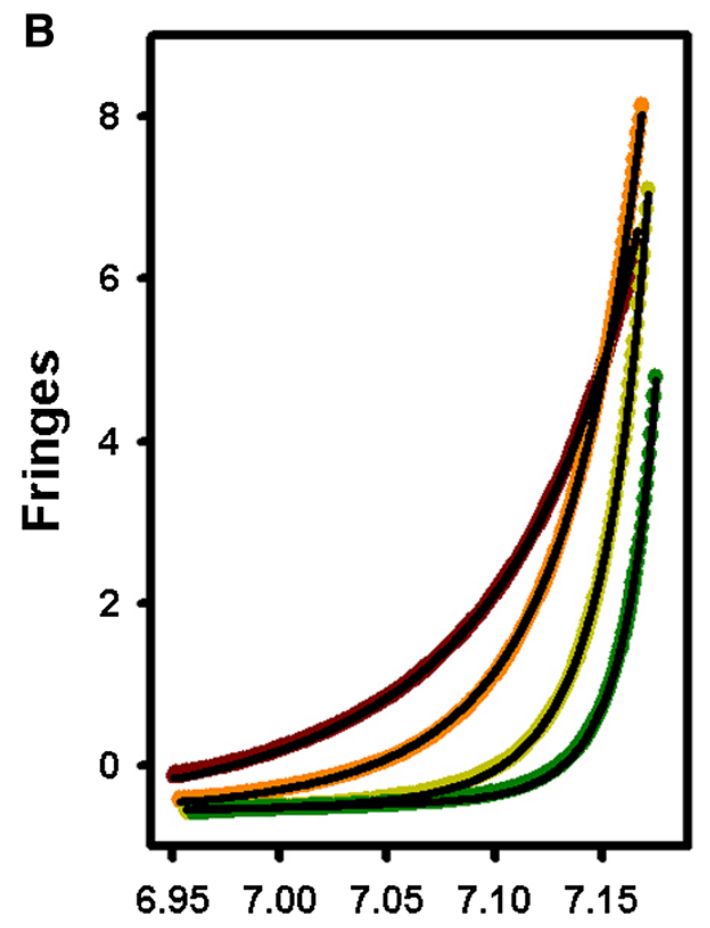

Radius (cm) 
Figure 4 Analytical ultracentrifugation of SIRPY, FabOX117 and SIRPY: FabOX117 complexes. (A) Sedimentation velocity distributions for SIRPY, FabOX117, a 2:1 and a 1:2 mixture of SIRPY: FabOX117. Initial sedimentation distributions were analysed in SEDFIT, whereas data were subsequently fitted in SEDANAL [22]. (B) Sedimentation equilibrium data of SIRPY: FabOX117 were obtained at 2:1, 1:1 and 1:2 stoichiometric ratios; for clarity, only the data at 2:1 ratio is shown. Sedimentation equilibrium was attained at $15000 \mathrm{rpm}$ (black), $20000 \mathrm{rpm}$ (red), $28000 \mathrm{rpm}$ (green) and 36000 rpm (yellow with black dots). Data were extracted with SEDFIT [22] and analysed in SEDPHAT [23]. A single species model did not fit the data. The data were then fitted to an $A+B \leftrightarrow C$ model where $A$ is SIRPY, $B$ is FabOX117 and $C$ is the 1:1 complex; this fit is represented by the solid black line going through each of the data traces.

then it is surprising that no 2:2 complex was observed at the concentration of $13.7 \mu \mathrm{M}$ used in the sedimentation velocity experiments. The results of the AUC analyses imply that self-association of SIRP $\gamma$ only occurs, if at all, at very high protein concentrations and therefore is unlikely to be physiologically relevant.

\section{Conclusions}

A cross-reactive anti-SIRP monoclonal antibody (OX117) has proved useful in crystallizing human SIRP $\gamma$ and subsequently obtaining the structure of the antibody: antigen complex. This has enabled the cross-reactive epitope to be mapped to the first and second domains of SIRPY distal from the CD47 ligand binding site on domain 1. The structure of the FabOX117: SIRPY complex has also revealed the potential for SIRPs to form head-to-head dimers through an interaction between the domains 1 and 2 on adjacent molecules in the crystal. However this interaction was not observed in solution, so its physiological significance is questionable. Comparison of the three domain structures of SIRPY and SIRP $\alpha$ showed that these receptors can adopt different overall conformations due to the flexibility of the linker between the first two domains. Given the sequence similarity between all members of the SIRP family it seems likely that this is a property shared by all of the receptors.

\section{Methods}

\section{Protein production}

SIRPY and the Fab fragment of the anti-SIRP monoclonal antibody, OX117 [3] (FabOX117), were expressed as recombinant proteins in mammalian cells. FabOX117 was produced by co-transfection of HEK 293 T cells (available from the American Type Culture Collection as CRL11268) with vectors encoding the heavy and light chain genes using a CompacT SelecT robotic system (The Automation Partnership, Royston, UK) [24]. The Fab fragment was purified from the cell culture media by nickel affinity chromatography followed by size exclusion chromato- graphy [15]. The extracellular region of SIRPY was expressed using the SIRP $\alpha$ leader sequence residues 1-30 (accession number CAA71403) followed by residues 29-347 of SIRPY (accession number NP_061026) with a C-terminal tag of STRHHHHHH using the pEE14 vector in CHO Lec3.2.8.1 cells [25] as previously described [12]. $\mathrm{N}$-glycosylation in these cells is arrested at the high mannose state enabling de-glycosylation by treatment with endoglycosidase H/F1. The SIRPY was purified from the cell culture media using nickel affinity chromatography followed by size exclusion chromatography [26]. The FabOX117 and SIRPY proteins were mixed in a 1:1 molar ratio and incubated overnight at $4^{\circ} \mathrm{C}$. The FabOX117: SIRP $\gamma$ complex was treated with Endo F1 to remove the N-linked glycans then purified by size exclusion chromatography.

\section{Crystallization and structure solution}

The FabOX117: SIRPY (16.8 mg/ml) complex was crystallized from $0.2 \mathrm{M}$ magnesium acetate tetrahydrate, 20\% w/v polyethylene glycol 3350 (Hampton PEG/Ion Screen \#25). Data were collected to $2.5 \AA$ resolution at Diamond Light Source beamline I03 from a single crystal. Diffraction images each of $1.0^{\circ}$ oscillation and $0.5 \mathrm{~s}$ exposure were recorded on a ADSC Quantum 315 CCD detector at a X-ray wavelength of $0.9763 \AA$. The crystal

\section{Table 2 X-ray data collection and refinement statistics}

\begin{tabular}{|c|c|}
\hline Data collection & \\
\hline X-ray source & Diamond 103 \\
\hline Wavelength $(\AA)$ & 0.97630 \\
\hline Space group & $P 2,2,2$ \\
\hline Unit cell $(\AA)$ & $a=140.4, b=174.2, c=81.7$ \\
\hline Resolution range $(\AA)$ & $30.0-2.50(2.59-2.50)$ \\
\hline Unique reflections & $71197(7000)$ \\
\hline Completeness (\%) & $100(100)$ \\
\hline Redundancy & $12.3(10.5)$ \\
\hline Average $/ / \sigma /$ & $14.9(2.1)$ \\
\hline$R_{\text {merge }}$ & $0.157(--)$ \\
\hline Refinement & \\
\hline Resolution range $(\AA)$ & $30.0-2.50$ \\
\hline No. of atoms (protein/other atoms) & $11212 / 323$ \\
\hline Rms bond length deviation $(\AA)$ & 0.008 \\
\hline Rms bond angle deviation $\left(^{\circ}\right)$ & 1.2 \\
\hline Mean B-factor (protein/other atoms $\left[\AA^{2}\right]$ ) & $38 / 54$ \\
\hline Residues in preferred regions (\%) & $1112(89.5)$ \\
\hline Residues in allowed regions (\%) & $128(10.3)$ \\
\hline Residues in disallowed regions (\%) & $3(0.2)$ \\
\hline
\end{tabular}

${ }^{\mathrm{a}} \mathrm{R}_{\text {work }}$ and $\mathrm{R}_{\text {free }}$ are defined by $\mathrm{R}=\Sigma_{\text {hk }}|| F_{\text {obs }}|-| F_{\text {calc }}|| \Sigma_{\text {hkl }}\left|F_{\text {obs }}\right|$, where $h, k, l$ are the indices of the reflections (used in refinement for $\mathrm{R}_{\text {work }} ; 5 \%$, not used in refinement, for $R_{\text {free }}$ ), $F_{\text {obs }}$ and $F_{\text {calc }}$ are the structure factors, deduced from measured intensities and calculated from the model, respectively. 
was soaked in a cryoprotectant solution containing $20 \%$ $(\mathrm{v} / \mathrm{v})$ glycerol and $80 \%(\mathrm{v} / \mathrm{v})$ crystallization reservoir solution for about $10 \mathrm{~s}$ before being plunged into liquid nitrogen and maintained at $100 \mathrm{~K}$ under a stream of nitrogen gas during data collection. Data were indexed, integrated and scaled using HKL2000/SCALEPACK [27]. The crystal belongs to a space group of $P 2_{1} 2_{1} 2$ with unit cell dimensions of $a=140.4 \AA, b=174.2 \AA$ and $c=81.7 \AA$. The solvent content is $54 \%$ by assuming 2 complexes in one crystallographic asymmetric unit.

The structure of the FabOX117: SIRPY complex was solved by molecular replacement using program MOLREP [28] and search models FabOX117 and SIRPa (PDB IDs: 3DIF and 2WNG). The orientations and positions of Fabs were readily determined, while the domains of SIRPY had to be found separately by using individual domains of SIRP $\alpha$ as search models. Structure refinement and model rebuilding were carried out with REFMAC [29] and COOT [30] respectively. Tight backbone and loose sidechain NCS restraints were applied throughout the refinement. Diffraction data and structure refinement statistics are shown in Table 2. Structural comparisons used SHP [31]. Structural images for figures were prepared with PyMOL (http://www.pymol.org/). The coordinates and structure factors have been deposited in the Protein Data Bank under accession number 4I2X.

\section{Analytical ultracentrifugation}

Analytical ultracentrifuge experiments were performed on a Beckman-Coulter Proteome Lab XL-I running version 5.8 of the data collection software. Data was obtained using both absorbance and interference optics. Sedimentation velocity data was obtained at $40000 \mathrm{rpm}$ in 2 channel meniscus-matching centrepiece cells (SpinAnalytical, NH, USA), while sedimentation equilibrium data was obtained at $15000,20000,28000$ and $36000 \mathrm{rpm}$ in 2 channel centrepieces (Beckman, USA).

Data were scanned every 2 hours until equilibrium had been reached, as determined by calculated RMSDs between successive scans using SEDFIT v14.1 and analysed using SEDPHAT. Loading concentrations were 1.0, 0.2, $0.1 \mathrm{mg} / \mathrm{ml}$ for Sirpy and FabOX117 alone. These corresponded to molar loading concentrations of 20, 4 and $2 \mu \mathrm{M}$ for Sirpy and 23, 4.2 and $2.1 \mu \mathrm{M}$ for FabOX117. For the mixtures, these were performed at a total concentration of $1 \mathrm{mg} / \mathrm{ml}$ at molar ratios of 1:2, 1:1 and 2:1, respectively. These corresponded to molar loading concentrations of $6.3 / 13.7 \mu \mathrm{M}, 10 / 10 \mu \mathrm{M}$ and $13.7 /$ $6.3 \mu \mathrm{M}$. The buffer used throughout was $20 \mathrm{mM}$ Tris$\mathrm{HCl}$ pH $7.5200 \mathrm{mM} \mathrm{NaCl}$. The solvent density and viscosity were calculated to be 1.0070 and 1.002 , respectively using SEDNTERP [32]. The molecular weight and partial specific volumes for FabOX117 was calculated using SEDNTERP to be $48380 \mathrm{Da}$ and $0.7281 \mathrm{~g} / \mathrm{ml}$, respectively. The corresponding values for SIRPy were calculated on the basis of an additional $1.8 \mathrm{kDa}$ of glycosylation to the protein sequence and found to be 52 $800 \mathrm{Da}$ and $0.7321 \mathrm{~g} / \mathrm{ml}$, respectively. All data were obtained at $20^{\circ} \mathrm{C}$.

\section{Data analysis of analytical ultracentrifuge data}

Sedimentation velocity data were analysed using SEDFIT v14.1, and sedimentation coefficients determined from the weight averaged integration of the peaks using the integration functions contained in the software. Corrected sedimentation coefficients ( $S_{20 \text {, w }}$ values) were noted for each loading concentration and used to extrapolate back to infinite dilution to obtain values for $S_{20 . w}^{0}$ for each species. Sedimentation equilibrium data were excised using SEDFIT v14.1 and then exported into SEDPHAT. Data were then fitted to a heterogeneous association $A+B \Leftrightarrow$ $\mathrm{AB}$, where $\mathrm{A}=$ FabOX117 (light and heavy chain) and $\mathrm{B}=$ SIRPY monomer. Errors in the determined dissociation constant were calculated within SEDPHAT using a Monte Carlo routine, and errors were quoted at the $95 \%$ confidence level.

\section{Competing interests}

The authors declare that they have no competing interest.

\section{Authors' contributions}

ANB and RJO initiated the study. JN, DH, NR, YZ produced the proteins, JN crystallized the complex, JR collected and processed the diffraction data, modeled and refined the structure. JR, ANB, and RJO analyzed the structure. DJS carried out AUC measurements and analyzed the data. JR, ANB, DIS, RJO and DJS wrote the paper. All authors read and approved the final manuscript.

\section{Acknowledgements}

The OPPF-UK is funded by the Medical Research Council and the Biotechnology and Biological Research Council. DIS is supported by the MRC, ANB and DH are funded by the MRC (grant ref; G9826026) and DJS is supported by the Science and Technology Facilities Council. The Wellcome Trust Centre for Human Genetics is supported by the Wellcome Trust (grant no.075491/Z/04). We thank the staff of beamline 103 at Diamond Light Source for help with data collection.

\section{Author details}

${ }^{1}$ Division of Structural Biology, Henry Wellcome Building for Genomic Medicine, University of Oxford, Roosevelt Drive, Oxford OX3 7BN, UK. ${ }^{2}$ OPPF-UK, The Research Complex at Harwell, Rutherford Appleton Laboratory, Harwell Oxford, Oxfordshire OX11 OFA, UK. ${ }^{3}$ The Research Complex at Harwell and ISIS Neutron and Muon source, Rutherford Appleton Laboratory, Harwell Oxford, Oxfordshire OX11 OFA, UK. ${ }^{4}$ The School of Biosciences, University of Nottingham, Sutton Bonington Campus, Sutton Bonington, Leicestershire LE12 5RD, UK. ${ }^{5}$ Sir William Dunn School of Pathology, University of Oxford, South Parks Road, Oxford OX1 3RE, UK. ${ }^{6}$ Diamond Light Sources, Harwell Science and Innovation Campus, Didcot OX11 ODE, UK.

Received: 6 December 2012 Accepted: 24 June 2013

Published: 4 July 2013

\section{References}

1. Barclay AN, Brown MH: The SIRP family of receptors and immune regulation. Nat Rev Immunol 2006, 6(6):457-464.

2. Barclay AN: Signal regulatory protein alpha (SIRPalpha)/CD47 interaction and function. Curr Opin Immunol 2009, 21(1):47-52. Epub 2009 Feb 2014. 
3. Brooke G, Holbrook JD, Brown MH, Barclay AN: Human lymphocytes interact directly with CD47 through a novel member of the signal regulatory protein (SIRP) family. J Immunol 2004, 173(4):2562-2570.

4. Piccio L, Vermi W, Boles KS, Fuchs A, Strader CA, Facchetti F, Cella M, Colonna M: Adhesion of human T cells to antigen-presenting cells through SIRPbeta2-CD47 interaction costimulates T-cell proliferation. Blood 2005, 105(6):2421-2427.

5. Van den Berg TK, Van Beek EM, Buhring HJ, Colonna M, Hamaguchi M, Howard CJ, Kasuga M, Liu Y, Matozaki T, Neel BG, et al: A nomenclature for signal regulatory protein family members. J Immunol 2005, 175(12):7788-7789.

6. Lee WY, Weber DA, Laur O, Severson EA, McCall I, Jen RP, Chin AC, Wu T, Gernert KM, Parkos CA: Novel structural determinants on SIRP alpha that mediate binding to CD47. J Immunol 2007, 179(11):7741-7750.

7. Liu Y, Tong Q, Zhou Y, Lee HW, Yang JJ, Buhring HJ, Chen YT, Ha B, Chen $C X$, Yang $Y$, et al: Functional elements on SIRPalpha IgV domain mediate cell surface binding to CD47. J Mol Biol 2007, 365(3):680-693.

8. Van den Berg TK, Yoder JA, Litman GW: On the origins of adaptive immunity: innate immune receptors join the tale. Trends Immunol 2004, 25(1):11-16.

9. Hatherley D, Harlos K, Dunlop DC, Stuart DI, Barclay AN: The structure of the macrophage signal regulatory protein alpha (SIRPalpha) inhibitory receptor reveals a binding face reminiscent of that used by $T$ cell receptors. J Biol Chem 2007, 282(19):14567-14575.

10. Nakaishi A, Hirose M, Yoshimura M, Oneyama C, Saito K, Kuki N, Matsuda M, Honma N, Ohnishi H, Matozaki T, et al: Structural insight into the specific interaction between murine SHPS-1/SIRP alpha and its ligand CD47. J Mol Biol 2008, 375(3):650-660.

11. Hatherley D, Graham SC, Turner J, Harlos K, Stuart DI, Barclay AN: Paired receptor specificity explained by structures of signal regulatory proteins alone and complexed with CD47. Mol Cell 2008, 31(2):266-277.

12. Hatherley D, Graham SC, Harlos K, Stuart DI, Barclay AN: Structure of signal-regulatory protein alpha: a link to antigen receptor evolution. J Biol Chem 2009, 284(39):26613-26619.

13. Kovari LC, Momany C, Rossmann MG: The use of antibody fragments for crystallization and structure determinations. Structure 1995, 3(12):1291-1293.

14. Rader C: Overview on concepts and applications of Fab antibody fragments. Curr Protoc Protein Sci 2009, 6(6):6-9.

15. Nettleship JE, Ren J, Rahman N, Berrow NS, Hatherley D, Barclay AN, Owens $\mathrm{RJ}$ : A pipeline for the production of antibody fragments for structural studies using transient expression in HEK 293T cells. Protein Expr Purif 2008, 62(1):83-89.

16. Krissinel E, Henrick K: Secondary-structure matching (SSM), a new tool for fast protein structure alignment in three dimensions. Acta Crystallogr D: Biol Crystallogr 2004, 60(Pt 12 Pt 1):2256-2268

17. Krissinel $\mathrm{E}$ : On the relationship between sequence and structure similarities in proteomics. Bioinformatics 2007, 23(6):717-723.

18. Lo Conte L, Chothia C, Janin J: The atomic structure of protein-protein recognition sites. J Mol Biol 1999, 285(5):2177-2198.

19. Brookes E, Demeler B, Rosano C, Rocco M: The implementation of SOMO (SOlution MOdeller) in the UltraScan analytical ultracentrifugation data analysis suite: enhanced capabilities allow the reliable hydrodynamic modeling of virtually any kind of biomacromolecule. Eur Biophys J 2010, 39:423-435.

20. Cann J: Interacting Macromolecules, Volume 12. New York, London: Academic; 1970

21. Nichol LaW DJ: Migration of Interacting Systems. Oxford: Oxford University Press; 1972

22. Schuck P: Size-distribution analysis of macromolecules by sedimentation velocity ultracentrifugation and lamm equation modeling. Biophys $J$ 2000, 78:1606-1619.

23. Vistica J, Dam J, Balbo A, Yikilmaz E, Mariuzza RA, Rouault TA, Schuck P: Sedimentation equilibrium analysis of protein interactions with global implicit mass conservation constraints and systematic noise decomposition. Anal Biochem 2004, 326:234-256.

24. Zhao Y, Bishop B, Clay JE, Lu W, Jones M, Daenke S, Siebold C, Stuart DI, Jones EY, Aricescu AR: Automation of large scale transient protein expression in mammalian cells. J Struct Biol 2011, 175(2):209-215.

25. Stanley P: Glycosylation mutants of animal cells. Annu Rev Genet 1984, 18:525-552.
26. Nettleship JE, Rahman-Huq N, Owens RJ: The production of glycoproteins by transient expression in mammalian cells. Methods Mol Biol 2009, 498:245-263.

27. Otwinowski ZMW: Processing of X-ray diffraction data collected in oscillation mode. Methods Enzymol 1997, 276:307-326.

28. Vagin A A, Teplyakov A: MOLREP: an automated program for molecular replacement. J App/ Cryst 1997, 30:1022-1025.

29. Murshudov GN, Vagin AA, Dodson EJ: Refinement of macromolecular structures by the maximum-likelihood method. Acta Crystallogr D: Biol Crystallogr 1997, 53(Pt 3):240-255.

30. Emsley P, Cowtan K: Coot: model-building tools for molecular graphics. Acta Crystallogr 2004, 60(Pt 12 Pt 1):2126-2132.

31. Stuart DI, Levine $M$, Muirhead $H$, Stammers DK: Crystal structure of cat muscle pyruvate kinase at a resolution of 2.6 A. J Mol Biol 1979, 134(1):109-142.

32. Laue TM, Shah BD, Ridgeway TM, Pelletier SL: Analytical Ultracentrifugation in Biochemistry and Polymer Science. In Royal Society of Chemistry. Edited by Harding S, Rowe A; 1992:90-125.

doi:10.1186/1472-6807-13-13

Cite this article as: Nettleship et al:: Crystal structure of signal regulatory protein gamma (SIRP $\gamma$ ) in complex with an antibody Fab fragment. BMC Structural Biology 2013 13:13.

\section{Submit your next manuscript to BioMed Central and take full advantage of:}

- Convenient online submission

- Thorough peer review

- No space constraints or color figure charges

- Immediate publication on acceptance

- Inclusion in PubMed, CAS, Scopus and Google Scholar

- Research which is freely available for redistribution 